This is a postprint version of the following published document:

A. Tapetado, J. Díaz-Álvarez, H. Miguélez, and C. Vázquez (2016). "Two-Color Pyrometer for Process Temperature Measurement During Machining". Lightwave Technology, Journal 34 (2016) 4, February, 15 pp. 1380 - 1386. Available in http://www.dx.doi.org/10.1109/JLT.2015.2513158 .

(C) 2016. IEEE. Personal use of this material is permitted. Permission from IEEE must be obtained for all other uses, in any current or future media, including reprinting/republishing this material for advertising or promotional purposes, creating new collective works, for resale or redistribution to servers or lists, or reuse of any copyrighted component of this work in other works. 


\title{
Two-Color Pyrometer for Process Temperature Measurement During Machining
}

\author{
Alberto Tapetado ${ }^{1}$, José Díaz- Álvarez², María Henar Miguélez ${ }^{3}$, \\ and Carmen Vázquez ${ }^{1}$, Senior Member, IEEE

\footnotetext{
1. Displays and Photonics Applications Group, Electronics Technology Department, Carlos III Uni-versity of Madrid, Legan. s 28911, Spain (e-mail: atapetad@ing.uc3m.es; cvazquez@ing.uc3m.es).

2. Aerospace Engineering Department, Carlos III University of Madrid, Leganés 28911, Spain (e-mail: jodiaz@ing.uc3m.es).

3. Mechanical Engineering Department, Carlos III University of Madrid, Leganés 28911, Spain (e-mail: mhmiguel@ing. uc3m.es).
}

\begin{abstract}
A fast fiber-optic two-color pyrometer operating on the optical communication bands is designed for temperature measurements in machining processes. Off-the-shelf low-loss fiber-optic demultiplexers and optoelectronics equipment are used in order to obtain a cost-effective sensing solution while reducing both the temperature measurement error and the minimum measurable temperature. The system is capable of measuring highly localized temperatures without using collimation lens. The designed pyrom-eter allows measuring temperature in the range from 300 to $650{ }^{\circ} \mathrm{C}$, achieving a full-scale temperature error as low as $4 \%$. Factors in-fluencing the temperature measurements are studied in order to identify the sensor limitations, such as a possible damage on the end of the optical fiber, the spectral loss attenuation and responsivity, or the distance between the fiber end and the target. Finally, this pyrometer is applied in a turning process, using a fiber-optic sensor embedded on a standard tool holder. Temperature measurements on the Inconel 718 are reported showing a good agreement with the simulations.
\end{abstract}

Keywords: Demultiplexer, fiber-optics, machining process, temperature measurement, two-color pyrometer, wavelength division multiplexing.

\section{Introcuction}

There is a clear trend in the aerospace industry towards reducing weight of the engine components and fuselage structure [1]. This trend has enable aircrafts to fly farther and faster and has made commercial flying more economical by allowing a larger aircraft capacity reducing the fuel consumption at the same time [2]. Achieving this goal has required the development of new materials with extra attributes in addition to light weight. For this reason, the use of superalloys plays a significant role in the development of the next-generation aircrafts. These materials also show excellent properties such as high strength at elevated temperatures, less fatigue sensitive and wear resistance. The ability to maintain these properties at elevated temperatures severely hinders the machinability of these alloys. The energy consumed by superalloys in a machining process is largely converted into heat and temperatures are high[3].

Temperature influences on tool wear reducing tool life and productivity. Therefore accurate temperature measurements in precise locations hold the key to improve the tool life and its surface integrity. The measurement of temperature has been reported using thermocouples [4] and infrared (IR) cameras [5]. The difficulty of installation between the cutting tool and the workpiece in these approaches [6] make them unsuitable for temperature measurements with the requirement of a specific sensing location.

Two-color fiber-optic pyrometer is a possible solution to overcome these constraints. It uses the ratio of optical powers at two spectral bands to implement a self-referencing technique in order to avoid the emissivity dependence of temperature as reported by Müller and Renz [7] and Thevenet et al. [8], among others [9], [10].
But some of these sensors use long wavelengths [7], [8], and free-space optics sensible to vibrations and with high insertion losses. This last disadvantage is compensated us-ing a cooling stage [7] to improve the detection sensitivity. All these aspects reduce the sensor robustness while increase the cost and the complexity of the system. In addition, the wave-length dependence losses of the photonics components used in several pyrometers are high. This is due, among others, to the use of photodetectors (PDs) with different responsivities at both selected wavelengths [9]. The use of optical fibers allows measuring temperatures in localized areas with a spatial resolution limited by the numerical aperture (NA) and dimensions of the fiber. In previous works, several authors propose measuring tem-peratures around $300{ }^{\circ} \mathrm{C}$ but using fiber diameters of 100 $\mu \mathrm{m}$ [8] and $450 \mu \mathrm{m}$ [9]. On the other hand, many authors propose the fiber-optic pyrometer application to high speed machining [7], however their experiments are not carried out in a machine tool during cutting. They are performed in a static block of al-loy preheated so they can not appreciate real peak values of temperature occurring close to the cutting area.

In this work, we propose a fiber-optic pyrometer to obtain temperature measurements of machined surfaces with a low temperature error by using an optimized design [11]. Commercial off-the-shelf optoelectronic components well established on wavelength division multiplexing (WDM) based optical communication networks are used. The characteristics of the optoelectronics and fiber-optic devices at the selected spectral bands are analyzed to achieve a temperature error reduction without thermal cooling and high spatial resolution. Finally, the experiments are carried out in a lathe, in cutting conditions similar 
to those imposed during current industrial operations, including models to validate the measurements.

\section{Theoretical background}

The spectral radiance of an object $(L)$ is given by Planck's law [12]:

$$
L(\lambda, T)=\frac{C_{1} \cdot \varepsilon(\lambda, T)}{\lambda^{5} \cdot\left(e^{\frac{C_{2}}{\lambda \cdot T}}-1\right)}
$$

where $\varepsilon$ is the emissivity of the measured object, $\lambda$ is the wavelength, $C_{1}$ and $C_{2}$ are the Planck's radiation constants, whose values are $1.191 \times 10^{8} \mathrm{~W} \cdot \mathrm{Sr}^{-1} \cdot \mu \mathrm{m}^{4} \cdot \mathrm{m}^{-2}$ and $1.439 \cdot 10^{4} \mu \mathrm{m} \cdot \mathrm{K}$, respectively, and $T$ is the absolute temperature of the body.

In a two-color fiber-optic pyrometer, the IR rays radiated from the object are accepted by an optical fiber and divided into two spectral bands. In the proposed design, this is accomplished using an optical filter. PDs measure the optical power at the different wavelength bands. Only rays inside the acceptance cone of the optical fiber are collected and transmitted, see Fig. 1. This acceptance angle $\left(\theta_{\mathrm{Max}}\right)$ depends on the optical fiber NA.

Setting location of the optical fiber is analyzed in [13]. The fiber end is vertically placed above the target surface at a distance $(t)$, see Fig. 1. We assume a target radius $\left(R_{T}\right)$ much greater than the spot radius $\left(R_{\mathrm{NA}}\right)$ projected by the fiber NA. Therefore, the current signal measured by the pyrometer can be expressed by:

$$
\begin{aligned}
I_{D}(T, \lambda)= & \int_{\lambda_{A}}^{\lambda_{B}} \frac{C_{1} \times R(\lambda) \times \operatorname{IL}(\lambda) \times \alpha(\lambda) \times \varepsilon(\lambda, T)}{\lambda^{5} \times\left(e^{\frac{C_{2}}{\lambda \times T}}-1\right)} \\
& \cdot \kappa(\lambda) \times d \lambda
\end{aligned}
$$

where $\kappa(\lambda)$ is the coupling loss given by:

$$
\kappa(\lambda)=\frac{\pi^{2} \cdot d_{\text {Core }}^{2}}{8} \cdot\left[1-\cos \left(2 \cdot \theta_{\text {Max }}(\lambda)\right)\right]
$$

being $\lambda_{A}$ and $\lambda_{B}$ the shortest and the longest wavelength values within the wavelength band provided by the optical filter, $R$ the PD responsivity, IL the insertion loss of the filter, $\alpha$ the fiber attenuation coefficient, and $d_{\text {Core }}$ the fiber core diameter.

From (2) and (3), the output signal ratio $(\gamma)$ between the current signals at both spectral bands is given by:

$$
\gamma=\frac{\int_{\lambda_{1, A}}^{\lambda_{1, B}} \frac{C_{1} \cdot R\left(\lambda_{1}\right) \cdot \operatorname{IL}\left(\lambda_{1}\right) \cdot \alpha\left(\lambda_{1}\right) \cdot \varepsilon\left(\lambda_{1}, T\right)}{\lambda_{1}^{5} \cdot\left(e^{\frac{C_{2}}{\lambda_{1} \cdot T}}-1\right)} \cdot \kappa\left(\lambda_{1}\right) \cdot d \lambda_{1}}{\int_{\lambda_{2, A}}^{\lambda_{2, B}} \frac{C_{1} \cdot R\left(\lambda_{2}\right) \cdot \operatorname{IL}\left(\lambda_{2}\right) \cdot \alpha\left(\lambda_{2}\right) \cdot \varepsilon\left(\lambda_{2}, T\right)}{\lambda_{2}^{5} \cdot\left(e^{\frac{C_{2}}{\lambda_{2} \cdot T}}-1\right)} \cdot \kappa\left(\lambda_{2}\right) \cdot d \lambda_{2}}
$$

where $\lambda_{i, A}$ and $\lambda_{i, B}$ correspond to the shortest and longest wavelengths of each spectral band $i$, with $i=1,2$.

If a two-color pyrometer working at single wavelengths channels $\lambda_{1}$ and $\lambda_{2}$ is considered, the measured ratio temperature $\left(T_{R}\right)$, as a modified version of equation reported in [7] to include loss ratio, is given by:

$$
T_{R}=\left[\frac{1}{T}+\frac{\ln \left(\varepsilon_{\mathrm{r}} \cdot \delta_{\mathrm{r}}\right)}{C_{2} \cdot\left(\frac{(-4) \cdot \Delta \lambda}{4 \cdot \lambda_{\mathrm{c}}^{2}-\Delta \lambda^{2}}\right)}\right]^{-1}
$$

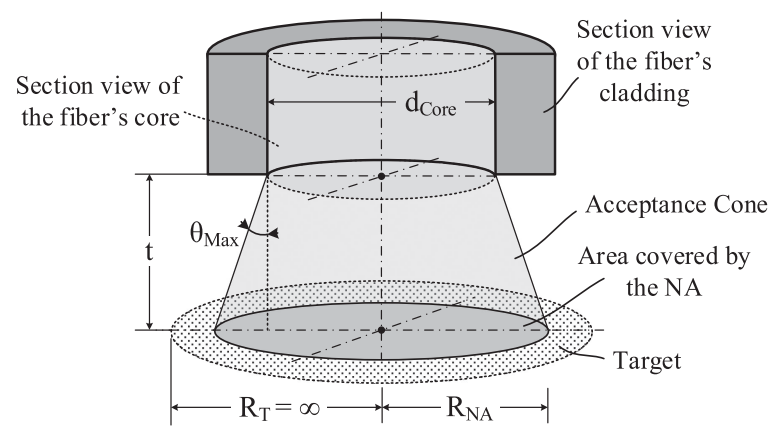

Fig. 1. Schematic of the optical fiber pyrometer measurement surface area.

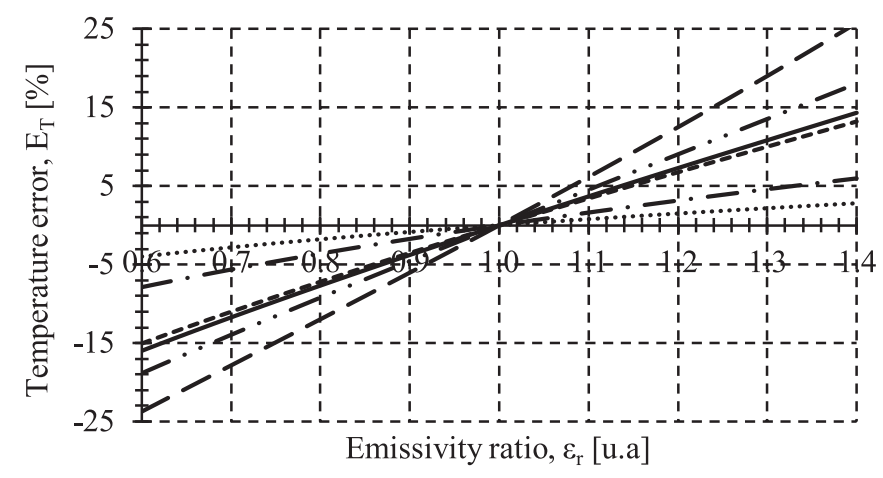

Fig. 2. Temperature error for different channels: $\cdots \Delta \lambda=0.9 \mu \mathrm{m}$ and $\lambda_{c}=$ $1.25 \mu \mathrm{m},-\Delta \lambda=0.3 \mu \mathrm{m}$ and $\lambda_{c}=1.4 \mu \mathrm{m},-\Delta \lambda=0.3 \mu \mathrm{m}$ and $\lambda_{c}=$ $1.45 \mu \mathrm{m},-\cdot-\Delta \lambda=0.6 \mu \mathrm{m}$ and $\lambda_{c}=1.4 \mu \mathrm{m},-\cdots-\Delta \lambda=0.24 \mu \mathrm{m}$ and $\lambda_{c}=1.43 \mu \mathrm{m},-\Delta \lambda=0.2 \mu \mathrm{m}$ and $\lambda_{c}=1.6 \mu \mathrm{m}$.

with

$$
\begin{aligned}
\varepsilon_{r} & =\frac{\varepsilon_{\lambda_{1}}}{\varepsilon_{\lambda_{2}}} \\
\delta_{r} & =\frac{R_{\lambda_{1}}}{R_{\lambda_{2}}} \cdot \frac{\alpha_{\lambda_{1}}}{\alpha_{\lambda_{2}}} \cdot \frac{L_{\lambda_{1}}}{L_{\lambda_{2}}} \cdot \frac{\kappa_{\lambda_{1}}}{\kappa_{\lambda_{2}}} \\
\lambda_{c} & =\frac{\lambda_{2}+\lambda_{1}}{2} \\
\Delta \lambda & =\lambda_{2}-\lambda_{1}
\end{aligned}
$$

where $T$ is the true temperature, $\lambda_{c}$ and $\Delta \lambda$ are the average central wavelength and the wavelength spacing of the single wavelength channels, respectively, $\delta_{r}$ is the loss ratio, and $\varepsilon_{r}$ is the surface emissivity ratio. And the relative temperature error $\left(E_{T}\right)$ is given by:

$$
E_{T}=\frac{\left(T_{R}-T\right) \cdot 100}{T} \quad[\%]
$$

\section{Pyrometer wavelength bands selection}

The primary error source when measuring the temperature in a pyrometer is that the grey-body behavior is not fulfilled. Especially, metals show very strong changes in their emissivities in the visible and near-IR range [14]. The temperature error causes by this behavior is analyzed using (5) to (10) for different wavelengths, see Fig. 2. A constant temperature of $500{ }^{\circ} \mathrm{C}$ and loss ratio of 1 are considered. 


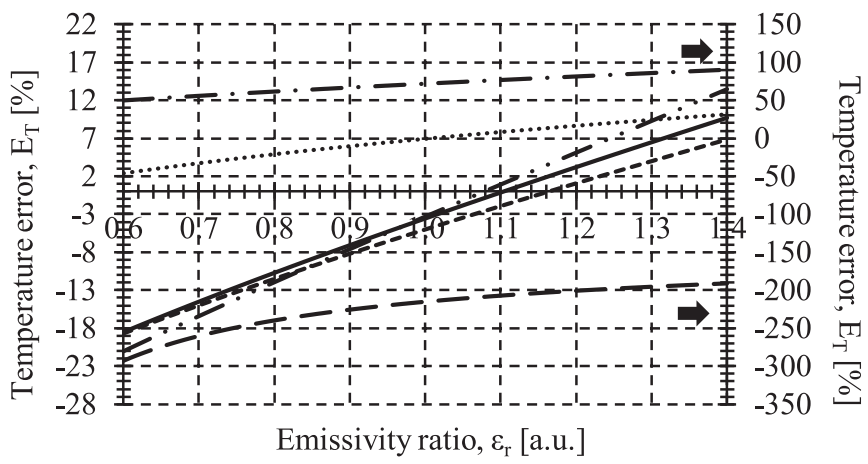

Fig. 3. Temperature error with InGaAs responsivity for different channels: (Left axis) $\cdots \Delta \lambda=0.9 \mu \mathrm{m}$ and $\lambda_{c}=1.25 \mu \mathrm{m},-\Delta \lambda=0.3 \mu \mathrm{m}$ and $\lambda_{c}=$ $1.4 \mu \mathrm{m},-\Delta \lambda=0.3 \mu \mathrm{m}$ and $\lambda_{c}=1.45 \mu \mathrm{m},-\cdots-\Delta \lambda=0.24 \mu \mathrm{m}$ and $\lambda_{c}=1.43 \mu \mathrm{m}$, (Right axis) $-\Delta \lambda=0.2 \mu \mathrm{m}$ and $\lambda_{c}=1.6 \mu \mathrm{m},-\cdot-\Delta \lambda=$ $0.6 \mu \mathrm{m}$ and $\lambda_{c}=1.4 \mu \mathrm{m}$.

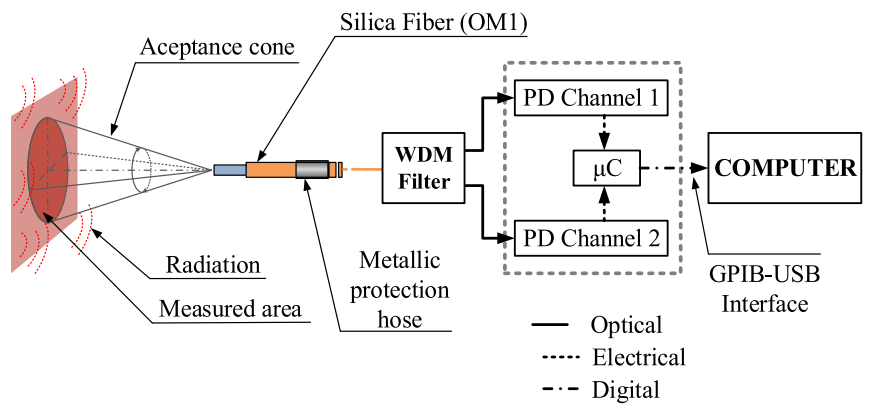

Fig. 4. Schematic of the pyrometer and the experimental set-up.

The temperature error decreases with the channel spacing. The separation between single wavelengths channels has to be large enough to minimize the temperature error and to allow the separation of both channels without overlapping. At the same time, they have to be close enough to ensure a constant emissivity [7].

Fig. 2 also shows that the temperature error becomes zero when changes in the emissivity are negligible. This is only valid when loss ratio equals 1 . But among others, the PD responsivity curve changes with wavelength. This effect is analyzed in Fig. 3. The temperature error increases around 3.3\%, using the InGaAs responsivity curve for an average central wavelength of $1.43 \mu \mathrm{m}$ and a wavelength spacing of $0.24 \mu \mathrm{m}$. Even higher values, around $72 \%$, are reached with other wavelength selections.

\section{Pyrometer desing and calibration Set-up}

The sensor is made of standard graded index glass optical fiber with $62.5 / 125 \mu \mathrm{m}$ core and cladding diameters. The fiber length is $0.5 \mathrm{~m}$. A low insertion loss WDM filter spatially splits the radiation collected by the optical fiber into two spectral bands centered at 1.3 and $1.55 \mu \mathrm{m}$, respectively. A dual InGaAs PDs with uniform responsivity at both wavelength bands is used. The schematic of the pyrometer as well as the experimental set-up are shown in Fig. 4.

The two-color fiber-optic pyrometer is calibrated using a dry block calibrator and a blackbody kit. The control unit ensures a maximum temperature stability and uncertainty of \pm 0.03 and

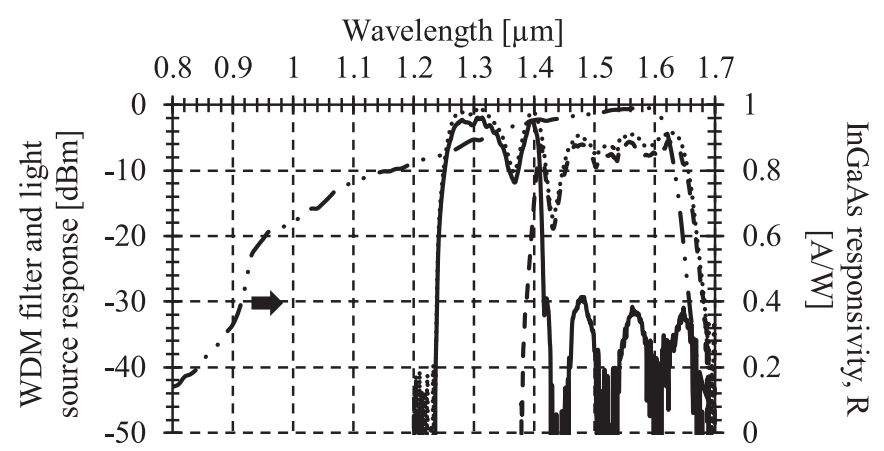

Fig. 5. (Left axis) WDM filter spectra: $-1.3 \mu \mathrm{m},-1.55 \mu \mathrm{m}, \cdots$ Input light source. (Right axis) $-\cdot \cdot-$ InGaAs responsivity.

$\pm 0.17{ }^{\circ} \mathrm{C}$, respectively, in the range from 50 to $650{ }^{\circ} \mathrm{C}$. The emissivity of the blackbody is greater than 0.99 .

\section{Experimental Results}

\section{A. Error Analysis}

The spectral response of the coupling loss, the fiber attenuation, the filter insertion losses and the PD responsivity are taken into account to evaluate the temperature error of the sensing scheme shown in Fig. 4.

As shown in (3), the coupling loss depends on the core diameter as well as the acceptance angle. The latter is given by:

$$
\sin \left(\theta_{\max }\right)=\sqrt{n_{\text {Core }}^{2}(\lambda)-n_{\text {Cladding }}^{2}(\lambda)}
$$

where $n_{\text {Core }}$ and $n_{\text {Cladding }}$ are the core and cladding refractive indices. The coupling ratio between the coupling loss at both spectral bands is calculated from (3) and (11), and using the Sellmeier dispersion relation [15]; a value of $0.014 \mathrm{~dB}$ is obtained.

Optical fiber attenuation coefficients at 1.3 and $1.55 \mu \mathrm{m}$ are 0.52 and $0.29 \mathrm{~dB} / \mathrm{km}$, respectively. They have a negligible impact in a $0.5 \mathrm{~m}$-long optical fiber.

Fig. 5 depicts the WDM filter wavelength spectra when a broadband light source is used. The filter loss ratio between both channels is $0.14 \mathrm{~dB}$.

Additionally, the influence of the PD responsivity is analyzed. Fig. 5 shows the InGaAs responsivity curve which shows a wavelength dependent loss of around $0.22 \mathrm{~dB}$ at the considered wavelengths bands.

Applying (5) to (10) the temperature error is calculated with the wavelength bands 1.3 and $1.55 \mu \mathrm{m}$ as a function of the emissivity ratio at $500{ }^{\circ} \mathrm{C}$, see Fig. 6 . In this figure, the wavelength dependence of the loss ratio is analyzed for each device individually. The overall effect is also shown.

The temperature error obtained considering the PD responsivity and the WDM filter insertion loss are $4.1 \%$ and $1.4 \%$, respectively, assuming an emissivity ratio equals to 1 . The contribution of the coupling loss and the fiber attenuation is practically negligible. Finally, the pyrometer temperature error due to the loss ratio is $5.5 \%$ for an emissivity ratio of 1, see Fig. 6 . The PD responsivities are equalized with the filter insertion loss spectra. This allows to have lower temperature errors at low 


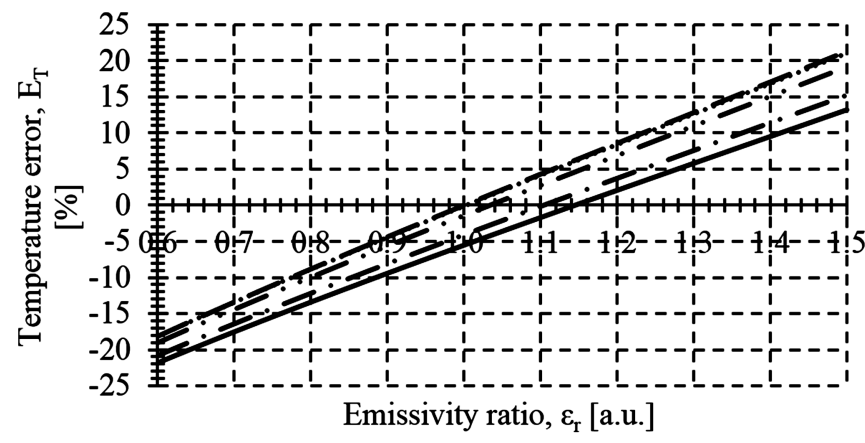

Fig. 6. Temperature error with: $-\cdots-$ WDM filter insertion loss, $\cdots$. Coupling loss, - - - PD responsivity, - Fiber attenuation, —- Two-color pyrometer (all loss ratio terms).

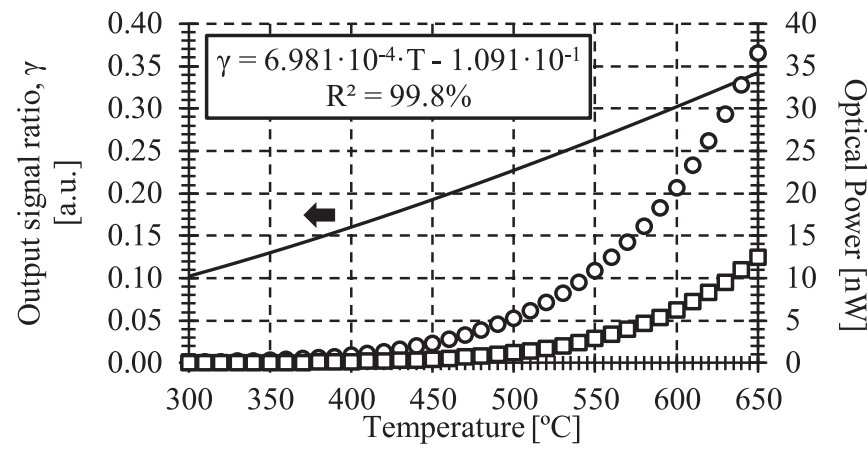

Fig. 7. Calibration curves for: ( $\square) 1.3 \mu \mathrm{m}$, (०) $1.55 \mu \mathrm{m},(-) 1.3 / 1.55 \mu \mathrm{m}$.

temperatures compared with other two-color pyrometer proposals [7], [9].

\section{B. Calibration Curves}

The radiant flux emitted by the blackbody has been characterized at both 1.3 and $1.55 \mu \mathrm{m}$ spectral bands, meanwhile the temperature of the blackbody surface changes from 300 to $650{ }^{\circ} \mathrm{C}$ at $10{ }^{\circ} \mathrm{C}$ intervals. A time interval of $45 \mathrm{~min}$ between each temperature measurement is set. The sample rate and the number of samples at each temperature and wavelength are fixed at $1 \mathrm{kHz}$ and $10 \mathrm{~S}$, respectively. The average optical power ratio at both spectral bands for each temperature is calculated from the mean of the samples. The results are shown in Fig. 7. The sensitivity obtained is $6.68 \times 10^{-4}{ }^{\circ} \mathrm{C}^{-1}$ and a full-scale temperature error of $4 \%$.

\section{Influence of Distance to Cutting Surface}

An important aspect in using optical fiber pyrometer in machining processes is to ensure an accurate positioning of the optical fiber end below the major cutting edge, see Fig. 8. The proposed system achieves a good spatial resolution while avoiding the use of lenses by placing the fiber end at a distance of a few hundred microns to the target (the fresh machined surface) and below the tool insert.

A set of measurements are carried out to evaluate the effect of the distance between the fiber end and the target during machining, as shown in Fig. 9. The same set-up described in the calibration section is used. The radiant flux emitted by the blackbody is measured at both spectral bands. The temperature of the

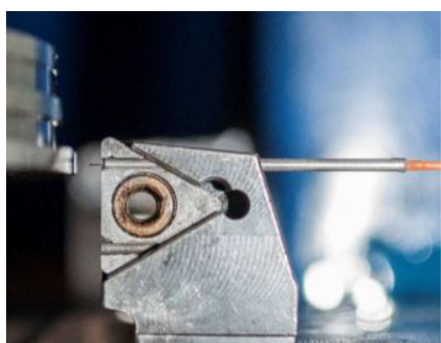

(a)

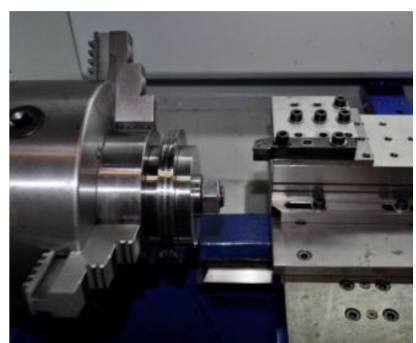

(b)
Fig. 8. (a) Visualization of the distance between the optical fiber and the workpiece (tool insert is removed). (b) Lathe with the machining tool.

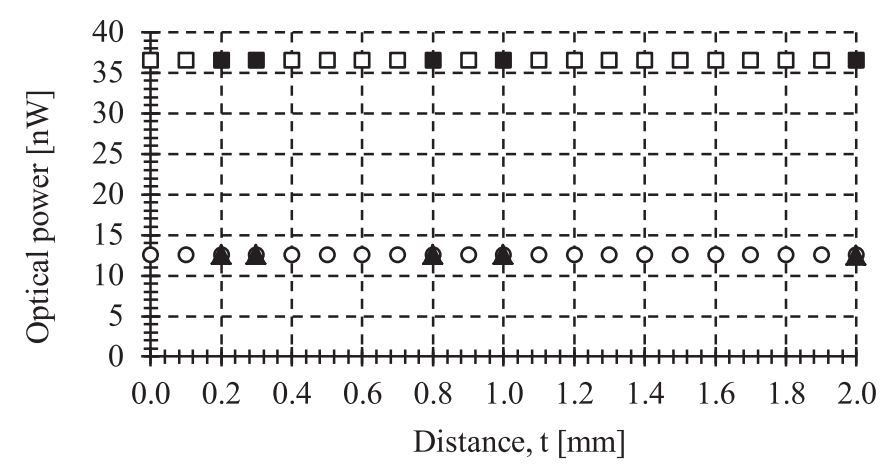

Fig. 9. Optical power versus distance for a wavelength of (circle) $1.3 \mu \mathrm{m}$ and (square) $1.55 \mu \mathrm{m}$ : (non-filled) Simulations, (filled) Measurements.

blackbody is fixed at $650{ }^{\circ} \mathrm{C}$ to ensure sufficient optical power to avoid the PD noise. Using calibrated washers, the distance between the blackbody and the fiber end is changed from 0.2 to $2 \mathrm{~mm}$ at different intervals. A set of ten measurements for each distance are taken.

The experimental results are compared with simulations. Using (2) and (3), the optical power received at each PD is calculated from a distance of 0 to $2 \mathrm{~mm}$, at $0.5 \mathrm{~mm}$ intervals. The wavelength dependence losses considered in the previous section are used in the pyrometer simulations.

Fig. 9 shows a deviation between the theoretical and experimental curves of 9.5 and $7.5 \mathrm{pW}$ for channels at 1.3 and $1.55 \mu \mathrm{m}$, respectively. Thus, considering sensor power accuracies below $3.4 \mathrm{pW}$. These power deviations are within the PD power accuracy. These results demonstrate that the experimental and theoretical values are in good agreement and there is not influence of the distance in our experiments.

\section{Influence of Optical Fiber Non-Perfect End}

An important issue when using optical fibers in an industrial machining environment is the possible damage of the fiber end. During machining, metal chips tend to detach from the interface between the cutting tool and the work piece. An example of a non-damage and a damage optical fiber end is illustrated in Fig. 10(a) and (b), respectively.

For a hot surface larger than the area covered by the fiber NA, the optical power measured by the PD is not dependent on the distance, see (2) and (3).

A set of 25 measurements of optical power at each spectral band are performed at a constant temperature of $650{ }^{\circ} \mathrm{C}$ with 
(a)

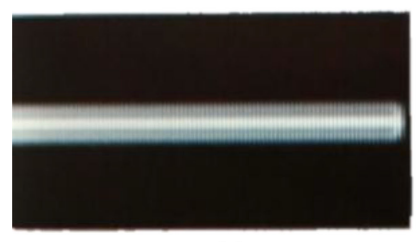

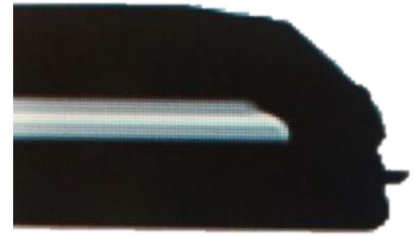

(b)
Fig. 10. Top view of the longitudinal section of the optical fiber. (a) Nondamage (b) Damage.

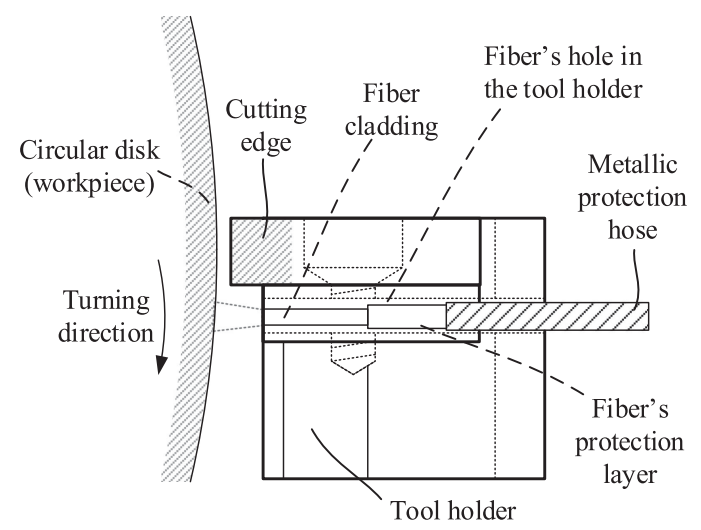

Fig. 11. Side view of the tool member with the optical fiber during the machining process.

a pyrometer made of fiber as the one shown in Fig. 10(b). The damage on an optical fiber end can be modelled by a small distance increment between the fiber end and the surface. The distance between the blackbody and the fiber is fixed at $0.3 \mathrm{~mm}$. There is no change in the measured optical power as expected.

\section{E. Temperature Measurements in Metal Cutting}

The machining experiments are conducted for continuous turning under two-dimensional orthogonal cutting conditions. The workpieces are shaped as a circular disk with a diameter and thickness of 120 and $2 \mathrm{~mm}$, respectively. The workpiece is located on a mandrel with a feed motion applied radially toward the axis of the disc. A modified tool holder is used to hold a carbide tool insert which has a rake and clearance angle of 0 and $7^{\circ}$, respectively. A scheme of the experimental set-up is shown in Fig. 11. The machining tests are performed at a linear cutting speed $\left(V_{c}\right)$, a depth of cut $(d)$ and a feed rate $(f)$ of $120 \mathrm{~mm} / \mathrm{min}, 2 \mathrm{~mm}$ and $0.05 \mathrm{~mm} / \mathrm{rev}$, respectively. No cutting fluids are used in the experiments. The cutting process takes less than $3 \mathrm{~s}$.

Using a computer software and the pyrometer set-up, a set of three measurements are carried out to perform a complete statistical analysis of the system in a real scenario, see Fig. 12.

As shown in Fig. 12, two different regions are observed during the temperature measurements. The first region occurs when the cutting tool starts to remove material from the workpiece. The radiant flux emitted by the machining surface is not great enough to be measured by the PDs. The PD noise is a limiting factor in order to measure the lower temperatures at the beginning of the cutting process. Along this region, forces increase quickly reaching a stationary regime after $0.75 \mathrm{~s}$. During this stationary

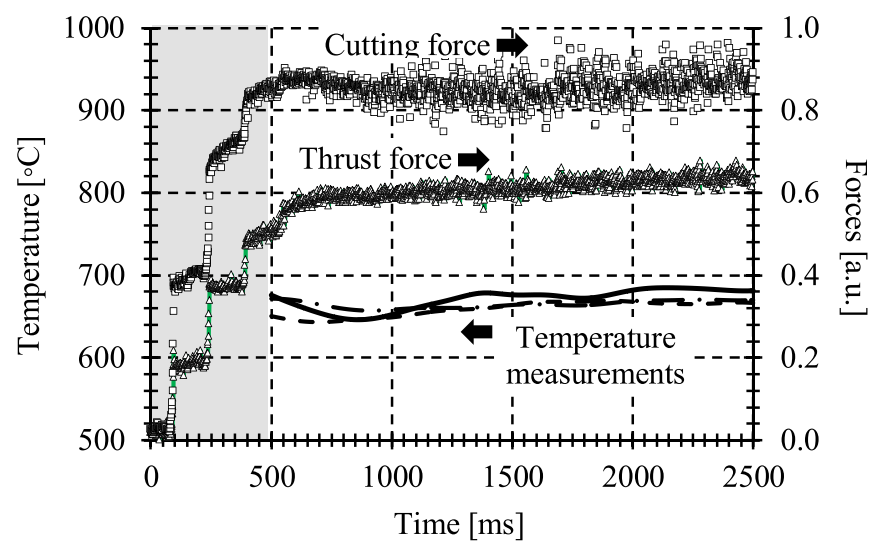

Fig. 12. Temperature measurements (discontinuous and continuous lines), and cutting (square dots) and thrust (triangle dots) force evolution when machining Inconel 718 with a carbide cutting tool: $V_{c}=120 \mathrm{~m} / \mathrm{min}, d=2 \mathrm{~mm}$, $f=0.05 \mathrm{~mm}$

regime, a maximum temperature of around $650{ }^{\circ} \mathrm{C}$ is obtained for a few seconds. Finally, using the three set of measurements, the repeatability in the stationary regime is analyzed. These results show that the two-color pyrometer is able to measure the temperature of $650{ }^{\circ} \mathrm{C}$ with an error of $14{ }^{\circ} \mathrm{C}$.

\section{Discussion}

Temperature measurement during cutting is extremely difficult due to the limited access to interesting measurement positions, especially locations close to the cutting edge. For this reason, there is no proper experimental technique suitable as a reference to compare with the designed pyrometer.

Numerical modeling based on finite element method is used to predict difficult measuring variables in the field of machining thermorresistant alloys [16]-[18]. The main disadvantage of using this technique is the high computational cost required to simulate the effect of subsequent passes of the cutting tool during the process on the machined surface. To overcome this problem, a combined analytical-numerical approach for the study of the steady-state cutting temperature is presented, as shown in Fig. 13. The numerical model allows estimating the amount of energy generated during the chip removal. On the other hand, the analytical model permits to calculate the temperature evolution in the workpiece due to the energy generated in the subsequent passes of the cutting tool.

In this section, both numerical and analytical models are described. The temperature prediction provided by the combined modeling scheme is presented.

\section{A. Numerical Modelling}

The numerical model, based on a Lagrangian approach including element deformation and further erosion, is carried out using a commercial finite element code (ABAQUS/Explicit). The model reproduces orthogonal turning tests using the same tool geometry and cutting parameters involved in the experimental work described in previous sections.

The sensitivity of the model with the mesh is checked and the element size is fixed equals to $3 \mu \mathrm{m}$, obtaining a tradeoff between accuracy and computation efficiency. 


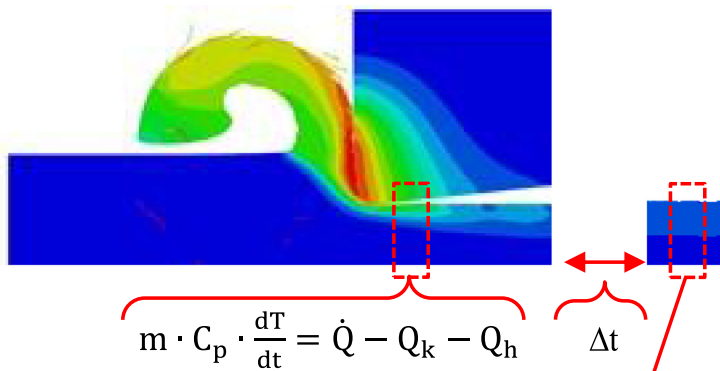

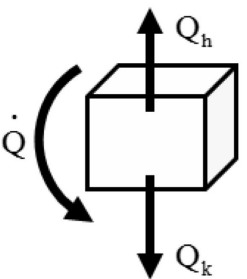

(a)

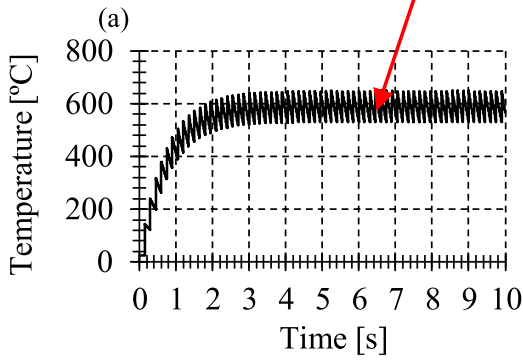

(b)
Fig. 13. (a) Temperature field after a machining pass and heat balance in a differential element located in the new created surface after the tool pass. (b) Temperature from the analytical model until the stationary state is achieved.

Element erosion required in order to avoid excessive distortion is based on a simple rule: when a critical level of the equivalent plastic strain is reached the elements are deleted. This rule is widely used in simulations of penetration problems. The critical strain is stated as 4 according to the mechanical phenomena involved during cutting, allowing realistic chip morphology.

The tool is assumed to behave rigid while the workpiece material exhibits an elastic viscoplastic, isotropic behavior following the $J_{2}$ flow theory (Mises yield criterion). The viscoplastic constitutive law is based on the Johnson-Cook equation [19]:

$$
\begin{aligned}
\sigma_{Y}= & \left(A+B \cdot \varepsilon_{p}^{n}\right) \cdot\left(1+C \cdot \ln \left(\frac{\dot{\varepsilon}_{p}}{\dot{\varepsilon}_{0}}\right)\right) \\
& \cdot\left(1-\left(\frac{T-T_{0}}{T_{m}-T_{0}}\right)^{m}\right)
\end{aligned}
$$

where $\sigma_{Y}$ is the tensile flow stress, $\varepsilon_{p}$ is the equivalent plastic strain, $\dot{\varepsilon}_{p}$ is the equivalent Mises plastic strain rate, $\dot{\varepsilon}_{0}$ is the effective plastic strain rate equals to $1.0 \mathrm{~s}^{-1}$, and $T$ is the true temperature. The alloy used in the experimental work, Inconel 718 alloy, is characterized with the parameters of the constitutive equation provided in [20].

It is assumed that the plastic work is mainly converted into heat with a Quinney-Taylor coefficient equals to $\beta=0.9$. The room temperature $\left(T_{0}\right)$ is assumed to be $25{ }^{\circ} \mathrm{C}$ for both the tool and the workpiece. The melting temperature $\left(T_{m}\right)$ is around $1627^{\circ} \mathrm{C}$.

A simple Coulomb law with a constant friction coefficient equals to 0.8 is used to simulate the tangential contact at the tool-chip interface. Despite of the simplicity of this formulation it is suitable to simulate adiabatic shearing at the primary cutting zone and sticking at the secondary cutting zone, phenomena governed by thermal softening as shown in [16], [18].
The geometrical and numerical characteristics of the model allow performing simulations of orthogonal cutting in a large range of cutting speeds, avoiding excessive mesh distortion and obtaining realistic level of cutting forces and chip morphology. The model is validated through the comparison between the predicted and the measured cutting forces obtained by means of a dynamometer used in the lathe during the turning tests. Errors around $7 \%$ in the cutting force and around $15 \%$ in the thrust force are obtained.

\section{B. Analytical Modeling}

The numerical model provides the amount of energy transferred to the workpiece in each pass of the cutting tool. The heat leads to a temperature rise in each pass of the tool. However, the heat loss due to convection and conduction should also be accounted, see Fig. 13(a). As in a single pass, it is impossible to reach the "stationary state" in the numerical model, the increment of energy per pass in a differential element is applied cyclically (being the period of the load equals to the time spent in each pass). Also convection and conduction are taken into account until a stationary state is achieved, see Fig. 13(b). Temperature predictions obtained with the analytical model agree with the measurements provided by the pyrometer, with an error of $1 \%$.

\section{Conclusion}

Temperature measurement in reduced areas located close to the cutting zone is a key challenge in order to ensure surface integrity and tool wear control. In this paper, a novel two-color fiber-optic pyrometer is developed and employed to measure temperature in a lathe, in cutting conditions similar to those imposed during current industrial operations with high spatial resolution.

It is demonstrated that the pyrometer temperature error is not only dependent on the selected wavelength bands but also on the spectral characteristics of the devices employed in the pyrometer set-up. A good selection of the pyrometer wavelength bands allows compensating the losses of each device, to minimize the pyrometer temperature error and to enhance the minimum measurable temperature. On the other hand, it is demonstrated that the measured temperature is independent of the distance between the fiber end and the target, and the possible damage of the sensing fiber end.

The pyrometer uses low-loss optical components designed for telecommunications purposes in order to reduce the temperature errors. The output power ratio at $\lambda_{1}=1.3 \mu \mathrm{m}$ and $\lambda_{2}=1.55 \mu \mathrm{m}$ performs a sensitivity of $6.981 \times 10^{-4}{ }^{\circ} \mathrm{C}^{-1}$, in a range from 300 to $600{ }^{\circ} \mathrm{C}$. The system shows a $4 \%$ full-scale temperature error.

Using the proposed two-color fiber-optic pyrometer and a lathe, the workpiece temperature in a high speed turning process is measured. In cutting of Inconel 718, the workpiece temperature is approximately $650{ }^{\circ} \mathrm{C}$ at cutting speed, cut depth and feed ratio of $120 \mathrm{~m} / \mathrm{min}, 2 \mathrm{~mm}$ and $0.05 \mathrm{~mm}$, respectively. The experimental results show a repeatability temperature error of $14^{\circ} \mathrm{C}$. This pyrometer makes it possible to measure the temperature history of the workpiece in turning. 
Numerical and analytical models are used to estimate the temperatures. The measured temperatures are in good agreement with the predicted values.

\section{Acknowledgments}

The authors would like to thank Dr. X. Soldani and E. García Ares for their collaboration in developing the cutting tool design and the positioning stage, respectively. This work was supported by the Spanish Ministry of Economíla $y$ Competitividad under Grants TEC2012-37983-C03-02, P2013/ MIT-2790, and DPI2014-56137-C2-2-R.

\section{References}

[1] J. C. Williams and E. A. Starke, "Progress in structural materials for aerospace systems," Acta Mater, vol. 51, no. 19, pp. 5775-5799, Nov. 2003.

[2] E. O. Ezugwu, "Key improvements in the machining of difficult-to-cut aerospace superalloys," Int. J. Mach. Tools Manuf., vol. 45, nos. 12/13, pp. 1353-1367, Oct. 2005.

[3] D. Dudzinski, A. Devillez, A. Moufki, D. Larrouquère, V. Zerrouki, and J. Vigneau, "A review of developments towards dry and high speed machining of Inconel 718 alloy," Int. J. Mach. Tools Manuf., vol. 44, no. 4, pp. 439-456, Mar. 2004.

[4] B. Alvelid, "Cutting temperature thermo-electrical measurements," Ann. CIRP, vol. 18, pp. 547-554, 1970.

[5] C. Dinc, I. Lazoglu, and A. Serpenguzel, "Analysis of thermal fields in orthogonal machining with infrared imaging," J. Mater. Process. Technol., vol. 198, nos. 1-3, pp. 147-154, Mar. 2008.

[6] A. Goyal, S. Dhiman, S. Kumar, and R. Sharma, "A study of experimental temperature measuring techniques used in metal cutting," Jordan J. Mech. Ind. Eng., vol. 8, no. 2, pp. 82-93, 2014.

[7] B. Müller and U. Renz, "Development of a fast fiber-optic two-color pyrometer for the temperature measurement of surfaces with varying emissivities," Rev. Sci. Instrum., vol. 72, no. 8, pp. 3366-3374, 2001.
[8] J. Thevenet, M. Siroux, and B. Desmet, "Measurements of brake disc surface temperature and emissivity by two-color pyrometry," Appl. Therm. Eng., vol. 30, nos. 6/7, pp. 753-759, May 2010.

[9] F. J. Madruga, D. A. F. Fernandez, and J. M. Lopez-Higuera, "Error estimation in a fiber-optic dual waveband ratio pyrometer," IEEE Sens. J., vol. 4, no. 3, pp. 288-293, Jun. 2004.

[10] A. Tapetado, C. Vázquez, X. Soldani, H. Miguélez, and D. S. Montero, "Temperature sensor based on fiber optic pyrometer in material removal processes," in Proc. 22nd Int. Conf. Opt. Fiber Sens., 2012, vol. 8421, pp. 84212V-1-84212V-4.

[11] C. Vázquez, A. Tapetado, H. Miguelez, and J. Díaz-Álvarez, "Pirometro de fibra optica a dos colores," ES P201530546, 2015.

[12] R. G. Driggers, C. Hoffman, and R. Driggers, Radiometry for Remote Sensing. New York, NY, USA: Taylor \& Francis, 2011.

[13] T. Ueda, A. Hosokawa, and A. Yamamoto, "Studies on temperature of abrasive grains in Grinding-Application of infrared radiation pyrometer," J. Eng. Ind., vol. 107, no. 2, pp. 127-133, 1985.

[14] F. M. White, Heat Transfer. Reading, MA, USA: Addison-Wesley, 1984

[15] J. W. Fleming, "Material dispersion in lightguide glasses," Electron. Lett., vol. 14 , no. 11 , pp. 326-328, 1978.

[16] J. Díaz-Álvarez, J. L. Cantero, H. Miguélez, and X. Soldani, "Numerical analysis of thermomechanical phenomena influencing tool wear in finishing turning of Inconel 718," Int. J. Mech. Sci., vol. 82, pp. 161-169, 2014.

[17] M. H. Miguélez, X. Soldani, and A. Molinari, "Analysis of adiabatic shear banding in orthogonal cutting of Ti alloy," Int. J. Mech. Sci., vol. 75, pp. 212-222, Oct. 2013.

[18] A. Molinari, X. Soldani, and M. H. Miguélez, "Adiabatic shear banding and scaling laws in chip formation with application to cutting of Ti-6Al4V," J. Mech. Phys. Solids, vol. 61, no. 11, pp. 2331-2359, Nov. 2013.

[19] G. R. Johnson and W. H. Cook, "A constitutive model and data for metals subjected to large strains, high strain rates and high temperatures," in Proc. 7th Int. Symp. Ballistics, 1983, vol. 21, pp. 541-547.

[20] T. Kobayashi, J. W. Simons, C. S. Brown, and D. A. Shockey, "Plastic flow behavior of Inconel 718 under dynamic shear loads," Int. J. Impact Eng., vol. 35, no. 5, pp. 389-396, May 2008. 